\title{
PREPARAÇÃO E CARACTERIZAÇÃO ESPECTROSCÓPICA DE COMPLEXOS DE BORO - UMA PROPOSTA PARA UMA PRÁTICA INTEGRADA DE QUÍMICA INORGÂNICA
}

\author{
Karl Eberhard Bessler*, Adriana Torres de Sousa, Sebastião de Souza Lemos, Fernanda Batista Gomes e Elizeu José de Souza \\ Instituto de Química, Universidade de Brasília, 70904-970 Brasília - DF, Brasil
}

Recebido em 5/10/09; aceito em 3/2/10; publicado na web em 10/6/10

\begin{abstract}
PREPARATION AND SPECTROSCOPIC CHARACTERIZATION OF BORON COMPLEXES - A PROPOSAL FOR AN INTEGRATED INORGANIC LABORATORY. As a proposal for an undergraduate second or third year inorganic laboratory course, the present paper describes the preparation of three representative boron complexes: potassium tetrafluoroborate, pyridoxin boron complex and potassium bis(oxalato)borate. The complexes are characterised by infrared and multinuclear magnetic resonance spectroscopy $\left({ }^{1} \mathrm{H},{ }^{11} \mathrm{~B}\right.$ and $\left.{ }^{19} \mathrm{~F}\right)$ where isotopic effects are demonstrated.
\end{abstract}

Keywords: boron complexes; instrumental methods; inorganic laboratory experiments.

\section{INTRODUÇÃO}

A química de coordenação não é um campo exclusivo dos elementos metálicos. Não metais como o boro ou silício, entre outros, também são capazes de formar complexos de coordenação, às vezes chamados de compostos "hipervalentes". ${ }^{1}$ Compostos moleculares de boro $\mathrm{BX}_{3}$ de geometria trigonal plana são deficientes em elétrons ou aceitadores de elétrons (ácidos de Lewis). A química desses compostos é dominada pelas reações com bases de Lewis, nas quais complexos tetraedricamente coordenados, neutros BX $\mathrm{L}$ ("adutos") ou aniônicos $\left[\mathrm{BX}_{4}\right]^{-}$, são obtidos. Alguns exemplos mais conhecidos de complexos aniônicos homolépticos são o tetrafluoroborato $\left[\mathrm{BF}_{4}\right]^{-}$, o boranato $\left[\mathrm{BH}_{4}\right]^{-}$e o tetrafenilborato $\left[\mathrm{B}\left(\mathrm{C}_{6} \mathrm{H}_{5}\right)_{4}\right]^{-}$. Até o ácido bórico $\mathrm{B}(\mathrm{OH})_{3}$ é hidratado em solução aquosa formando o ânion $\left[\mathrm{B}(\mathrm{OH})_{4}\right]^{-}$ de acordo com a seguinte reação de equilíbrio:

$$
\mathrm{B}(\mathrm{OH})_{3}+2 \mathrm{H}_{2} \mathrm{O} \rightleftarrows \mathrm{H}_{3} \mathrm{O}^{+}+\left[\mathrm{B}(\mathrm{OH})_{4}\right]^{-}
$$

Uma grande variedade de quelatocomplexos tetraédricos de boro é conhecida e pode ser classificada da seguinte maneira: Monoquelatos $\mathrm{LBX}_{2}$ envolvendo um ligante quelante monoaniônico $\mathrm{L}$ como $\beta$-dicetonato, e ânions simples $\mathrm{X}$ como $\mathrm{F}, \mathrm{OH}$, alquila, etc.; ${ }^{1,2}$ bis-quelatos catiônicos $\left[\mathrm{BL}_{2}\right]^{+}$, neutros [BLL'] ou aniônicos $\left[\mathrm{BL}_{2}\right]^{-}$ com ligantes quelantes monoaniônicos $\mathrm{L}$ como $\beta$-dicetonatos, e dianiônicos L' como oxatato, malonato etc. ${ }^{1-4}$ Observa-se que a química de coordenação do boro, apesar de abordar aspectos didáticos fundamentais, tem sido raramente explorada no ensino experimental.

O boro natural é composto por dois isótopos estáveis, ${ }^{11} \mathrm{~B}$ e ${ }^{10} \mathrm{~B}$ com a abundância de 80,22 e 19,78\%, respectivamente (percentual atômico). A elevada abundância de ambos os isótopos e a considerável razão de massa ${ }^{10} \mathrm{~B} /{ }^{11} \mathrm{~B}$ viabilizam a observação do efeito isotópico de massa nos espectros vibracionais de compostos de boro. As frequências daqueles modos vibracionais, nas quais o átomo de boro é deslocado (vibrações antissimétricas $-v_{\mathrm{as}}$ ) apresentam consideráveis desdobramentos em dois componentes..${ }^{5,6}$ Por exemplo, as frequências de vibração de estiramento antissimétrica $\left(v_{a s} B_{4}\right)$ correspondem a 1034 e $1083 \mathrm{~cm}^{-1}$ para ${ }^{11} \mathrm{BF}_{4}^{-} \mathrm{e}^{10} \mathrm{BF}_{4}^{-}$, respectivamente.

A espectroscopia de ressonância magnética nuclear (RMN) é considerada uma ferramenta instrumental versátil, pois fornece informações estruturais inclusive de sistemas complexos. Quase todos os elementos

\footnotetext{
*e-mail: bessler@unb.br
}

químicos possuem um ou mais núcleos isotópicos estáveis com spin nuclear diferente de zero $(\mathrm{I} \neq 0)$, sendo, portanto, susceptíveis à ressonância magnética nuclear. Porém, experimentos didáticos envolvendo a espectroscopia de RMN são geralmente dedicados às disciplinas de química orgânica, explorando quase exclusivamente os núcleos ${ }^{1} \mathrm{H}$ e ${ }^{13} \mathrm{C}$. Apesar da facilidade na obtenção de espectros de RMN de boro, na bibliografia mais recente de ensino de química experimental, apenas um trabalho foi encontrado que aplica a técnica de RMN aos compostos de boro $\left({ }^{1} \mathrm{H} \mathrm{RMN}\right.$ de $\left.\mathrm{NaBH}_{4}\right){ }^{7}$ Ambos os núcleos do boro natural (spin nuclear $\mathrm{I}=3$ para ${ }^{10} \mathrm{~B}$ e I $=3 / 2$ para ${ }^{11} \mathrm{~B}$ ) são ativos na $\mathrm{RMN}$. O núcleo mais abundante ${ }^{11} \mathrm{~B}$ é preferível para estudos de $\mathrm{RMN}$, pois sua receptividade é trinta e quatro vezes maior que a do ${ }^{10} \mathrm{~B} .{ }^{8}$ Devido às suas características espectroscópicas favoráveis, um espectro de RMN ${ }^{11} \mathrm{~B}$, de uma solução suficientemente concentrada, pode ser obtido, em menos de 1 min em um espectrômetro moderno.

O flúor é um elemento monoisotópico $\left({ }^{19} \mathrm{~F}\right)$ e apresenta spin nuclear meio $(\mathrm{I}=1 / 2)$. $\mathrm{O}$ isótopo exerce um deslocamento químico induzido no núcleo de ${ }^{19} \mathrm{~F}\left[{ }^{1} \Delta^{10 / 11}(\mathrm{~B}-\mathrm{F})\right] .{ }^{9}$ Os espectros de RMN de ${ }^{19} \mathrm{~F}$ do complexo $\left[\mathrm{BF}_{4}\right]^{-}$apresentam resolução suficiente para permitir a discriminação entre $\left[{ }^{11} \mathrm{BF}_{4}\right]^{-}$e $\left[{ }^{10} \mathrm{BF}_{4}\right]^{-}$, relatado inclusive em espectros obtidos em um espectrômetro de baixo campo $\left(56,4 \mathrm{MHz}\right.$ para $\left.{ }^{19} \mathrm{~F}\right) .{ }^{10}$

Uma vantagem da aplicação da RMN para a finalidade proposta nesse trabalho é que podemos utilizar a mesma solução de $\mathrm{K}\left[\mathrm{BF}_{4}\right]$ em $\mathrm{D}_{2} \mathrm{O}$ para obtenção dos espectros tanto de ${ }^{19} \mathrm{~F}$ quanto de ${ }^{11} \mathrm{~B}$. É possível a obtenção desses espectros inclusive em $\mathrm{H}_{2} \mathrm{O}$, especialmente no caso de RMN de ${ }^{11} \mathrm{~B}$ onde os picos são naturalmente mais largos em comparação com aqueles do ${ }^{19} \mathrm{~F}$. Isso se deve ao fato de que os espectrômetros modernos de RMN apresentam elevada estabilidade do campo magnético $\left(\mathrm{B}_{0}\right)$ e permitem a rápida obtenção de espectros mediante aplicação de pulsos de radiofrequência $\left(\mathrm{B}_{1}\right)$ e posterior aplicação de transformada de Fourier.

No presente conjunto de experimentos são preparados três complexos de boro e caracterizados por meio de espectroscopia no infravermelho e espectroscopia de ressonância magnética multinuclear $\left({ }^{1} \mathrm{H},{ }^{11} \mathrm{~B} \mathrm{e}{ }^{19} \mathrm{~F}\right)$. Os efeitos isotópicos são demonstrados nos espectros tanto de infravermelho quanto de RMN. Os principais objetivos de nosso trabalho foram incluir exemplos da química do boro em práticas de química inorgânica, incentivar o emprego da ressonância magnética multinuclear no ensino experimental e demonstrar efeitos isotópicos por meio de métodos espectroscópicos.

Os tetrafluoroboratos são os complexos mais estáveis do boro, não sofrendo decomposição hidrolítica em meio aquoso. Um aspecto 
interessante é que o $\left.\mathrm{K}_{\left[\mathrm{BF}_{4}\right]}\right]$ representa um dos raros exemplos de sais de potássio pouco solúveis em água, enquanto o $\mathrm{Na}\left[\mathrm{BF}_{4}\right]$ apresenta boa solubilidade em água. Tetrafluoroboratos podem ser preparados convenientemente pela reação de ácido bórico com ácido fluorídrico aquoso e neutralização subsequente do ácido tetrafluorobórico com uma base apropriada, ${ }^{11}$ conforme as reações 2 e 3:

$$
\begin{aligned}
& \mathrm{H}_{3} \mathrm{BO}_{3}+4 \mathrm{HF} \rightarrow \mathrm{HBF}_{4}+3 \mathrm{H}_{2} \mathrm{O} \\
& \mathrm{HBF}_{4}+\mathrm{NaOH} \rightarrow \mathrm{NaBF}_{4}+\mathrm{H}_{2} \mathrm{O}
\end{aligned}
$$

Um complexo de boro com piridoxina (vitamina $\mathrm{B}_{6}$ ) é obtido a partir de ácido bórico e piridoxina em solução aquosa. ${ }^{12} \mathrm{~A}$ estrutura molecular corresponde a um bis-quelato neutro, no qual os dois ligantes piridoxínicos coordenam por meio de seus grupos fenólicos desprotonatos e os átomos de oxigênio hidroximetílicos adjacentes (Esquema 1). Em um dos ligantes de piridoxina o nitrogênio piridínico encontra-se protonado levando a uma estrutura betaínica ou "zwitteriônica". ${ }^{13} \mathrm{O}$ complexo cristaliza facilmente a partir da solução aquosa em forma de um mono-hidrato, o qual foi sugerido para isolamento e purificação da piridoxina. ${ }^{14}$

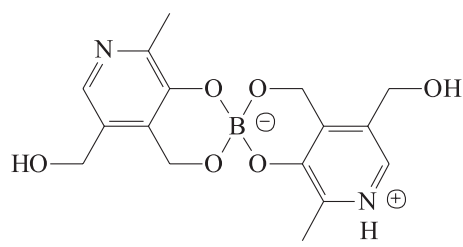

Esquema 1. Estrutura molecular do boro-complexo da piridoxina

O ácido oxálico é um excelente agente complexante. Oxalatocomplexos de uma ampla variedade de cátions metálicos são bem conhecidos. Oxalatoboratos não podem ser obtidos em meio aquoso devido a sua sensibilidade hidrolítica. Eles são preparados convenientemente a partir de ácido bórico, ácido oxálico e uma base apropriada, ou um oxalato, em tolueno sob refluxo com separação contínua da água de reação (método de Dean Stark), ${ }^{4}$ como, por exemplo, na reação 4 ou 5:

$$
2 \mathrm{H}_{3} \mathrm{BO}_{3}+4 \mathrm{H}_{2} \mathrm{C}_{2} \mathrm{O}_{4}+\mathrm{K}_{2} \mathrm{CO}_{3} \rightarrow 2 \mathrm{~K}\left[\mathrm{~B}\left(\mathrm{C}_{2} \mathrm{O}_{4}\right)_{2}\right]+7 \mathrm{H}_{2} \mathrm{O}+\mathrm{CO}_{2}
$$

ou

$$
2 \mathrm{H}_{3} \mathrm{BO}_{3}+3 \mathrm{H}_{2} \mathrm{C}_{2} \mathrm{O}_{4}+\mathrm{K}_{2} \mathrm{C}_{2} \mathrm{O}_{4} \rightarrow 2 \mathrm{~K}\left[\mathrm{~B}\left(\mathrm{C}_{2} \mathrm{O}_{4}\right)_{2}\right]+6 \mathrm{H}_{2} \mathrm{O}
$$

\section{PARTE EXPERIMENTAL}

\section{Equipamentos}

Os espectros de infravermelho podem ser registrados em um espectrômetro com varredura entre 400 e $4000 \mathrm{~cm}^{-1}$. Os espectros de RMN foram feitos em um espectrômetro com campo magnético de 7,05 T $\left(300,07 \mathrm{MHz}\right.$ para ${ }^{1} \mathrm{H}, 282,31 \mathrm{MHz}$ para ${ }^{19} \mathrm{~F}$ e $96,27 \mathrm{MHz}$ para ${ }^{11} \mathrm{~B}$ ), equipado com uma sonda de $5 \mathrm{~mm}$, sintonizável para o núcleo X. Entretanto, esse estudo pode ser conduzido em outra configuração de espectrômetro disponível, desde que seja possível sintonizar o núcleo de interesse.

\section{Preparação de tetrafluoroborato de potássio}

Atenção! O ácido fluorídrico é uma substância muito agressiva e altamente tóxica. Utilize luvas para prevenir o contato com a pele. Realize o procedimento dentro de uma capela de exaustão para evitar a inalação dos vapores de HF.

$O$ ácido fluorídrico ataca o vidro, portanto, devem ser empregados recipientes de polipropileno ou polietileno.

10 mL de ácido fluorídrico concentrado (40\%) são medidos em um cilindro graduado de polipropileno e transferidos a um béquer de polipropileno de $50 \mathrm{~mL}$. Adiciona-se em pequenas porções 3,1 g (50 mmol) de ácido bórico, agitando a mistura até a dissolução completa do ácido bórico. Acrescentam-se algumas gotas de solução de fenolftaleína.

Cuidado, reação exotérmica! A solução é neutralizada adicionando-se gota a gota, sob agitação, uma solução de hidróxido de potássio a $25 \%$ (cerca de $10 \mathrm{~mL}$ ) até que a solução adquira uma cor rosa permanente. Após esfriar a solução sobre um banho de gelo durante 30 min o produto sólido é filtrado a vácuo, lavado sobre o filtro com muito pouco de água gelada e guardado em um dessecador, carregado com hidróxido de sódio, durante $12 \mathrm{~h}$. O produto seco é pesado para o cálculo do rendimento (rendimento médio obtido $\mathrm{KBF}_{4}$ : 90 a $95 \%$ ).

Registra-se um espectro de infravermelho (em pastilha de $\mathrm{KBr}$ ) e compara-se o mesmo com o espectro de referência (Figura 1). Devido à simetria tetraédrica do íon $\mathrm{BF}_{4}{ }^{-}$observam-se apenas duas bandas vibracionais, ambas com o respectivo desdobramento isotópico. A primeira, mais intensa $\left(1035 / 1083 \mathrm{~cm}^{-1}\right)$ corresponde ao estiramento antissimétrico $v_{\mathrm{as}} \mathrm{BF}_{4}$; a outra $\left(522 / 534 \mathrm{~cm}^{-1}\right)$ é atribuída à deformação angular $\delta_{\text {as }} \mathrm{BF}_{4}$. $^{\text {as }}$

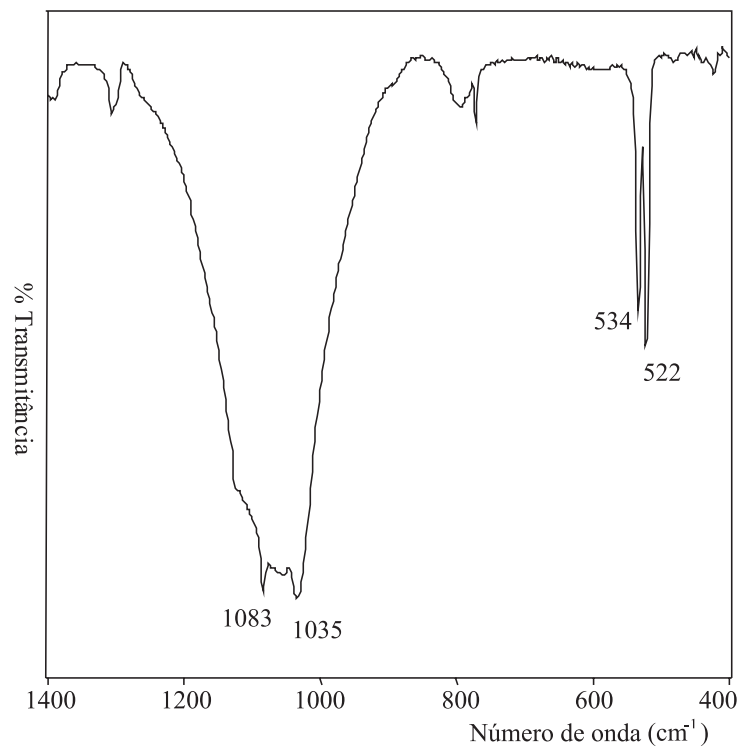

Figura 1. Espectro de infravermelho do $\mathrm{KBF}_{4}$

Para a obtenção dos espectros de RMN, é preparada uma solução de $10 \mathrm{mg}$ do $\mathrm{KBF}_{4}$ em $1 \mathrm{~mL}$ de água.

Adquire-se um espectro de RMN do núcleo de ${ }^{11} \mathrm{~B}$. Um único pico deve ser observado em $\delta=-1,2 \mathrm{ppm}$, referenciado externamente com $\mathrm{BF}_{3} \times \mathrm{OEt}_{2} 15 \%(\mathrm{v} / \mathrm{v})$ em $\mathrm{CDCl}_{3}(\delta=0)$, confrontado contra uma solução saturada de $\mathrm{H}_{3} \mathrm{BO}_{3}$ em $\mathrm{D}_{2} \mathrm{O}(\delta=19,6)$ contida em um capilar. Se eventualmente mais sinais forem detectados, correspondem à presença de outros compostos de boro como impurezas. As eventuais impurezas podem ser identificadas com ajuda de valores de referência $\delta{ }^{11} \mathrm{~B} .{ }^{15}$

Adquire-se um espectro de RMN do núcleo ${ }^{19} \mathrm{~F}$. O sinal de ressonância do íon $\mathrm{BF}_{4}{ }^{-}$é observado em $\delta=-150 \mathrm{ppm}$, referenciado externamente com $\mathrm{CCl}_{3} \mathrm{~F},(\delta=0)$, confrontado com uma solução de $\mathrm{CF}_{3} \mathrm{COOH} 0,5 \mathrm{~mol} / \mathrm{L} \mathrm{em} \mathrm{D} \mathrm{O}(\delta=-75,6)$. Quando o espectro é obtido em alta resolução, observa-se um desdobramento do sinal de $\mathrm{BF}_{4}^{-}$em 
dois componentes com as intensidades relativas de 1:4. Explique a origem desse fenômeno (efeito isotópico induzido). ${ }^{9}$ Se eventualmente mais sinais forem detectados, correspondem à presença de outras espécies contendo flúor como impurezas. Esses componentes podem ser identificados consultando-se os valores de referência $\delta{ }^{19} \mathrm{~F}$; no passado, os valores de deslocamento químico foram referenciados ao $\mathrm{CF}_{3} \mathrm{COOH}(\delta=0){ }^{16,17}$ Espécies do tipo $\left[\mathrm{BF}_{3} \mathrm{~A}\right]^{-}$onde A corresponde a um ânion monovalente são facilmente identificadas devido ao acoplamento com os isótopos de boro.

\section{Isolamento de piridoxina em forma do boro-complexo a partir de comprimidos de vitamina $B_{6}$}

Em um exemplo típico, o fabricante forneceu a seguinte informação referente à composição dos comprimidos de vitamina $\mathrm{B}_{6} \mathrm{ad}-$ quiridos na drogaria: cada comprimido contém $100 \mathrm{mg}$ de piridoxina cloridrato além de outros ingredientes.

Uma quantidade de comprimidos de vitamina $\mathrm{B}_{6}$ correspondente a um conteúdo total de $1 \mathrm{~g}$ (5 mmol) de piridoxina cloridrato é triturada em gral de porcelana. O pó é transferido para um béquer e agitado com 30 mL de uma solução de amônia a 10\% (a amônia é necessária para neutralizar o cloridrato). A mistura é filtrada e o resíduo é lavado sobre o filtro com $10 \mathrm{~mL}$ de água. Ao filtrado, $155 \mathrm{mg}(2,5 \mathrm{mmol})$ de ácido bórico são adicionados e a solução é evaporada em banhomaria até o volume de $10 \mathrm{~mL}$ e guardada em um lugar frio durante a noite. Os cristais formados são retirados e secos sobre papel de filtro. O produto seco é pesado para o cálculo do rendimento (rendimento médio obtido $\mathrm{C}_{16} \mathrm{H}_{19} \mathrm{BN}_{2} \mathrm{O}_{6} \cdot \mathrm{H}_{2} \mathrm{O}: 30$ a $40 \%$, baseado na informação do fornecedor dos comprimidos).

Registra-se um espectro de infravermelho (em pastilha de $\mathrm{KBr}$ ) e compara-se o mesmo com o espectro de referência (Figura 2). Devido à complexidade da estrutura molecular, o espectro é bastante complicado. A banda mais intensa em $1100 \mathrm{~cm}^{-1}$ pode ser atribuída à vibração de estiramento antissimétrica $\mathrm{v}_{\mathrm{as}} \mathrm{BO}_{4}$ com o componente do isótopo ${ }^{10} \mathrm{~B}$ em $1119 \mathrm{~cm}^{-1}$.

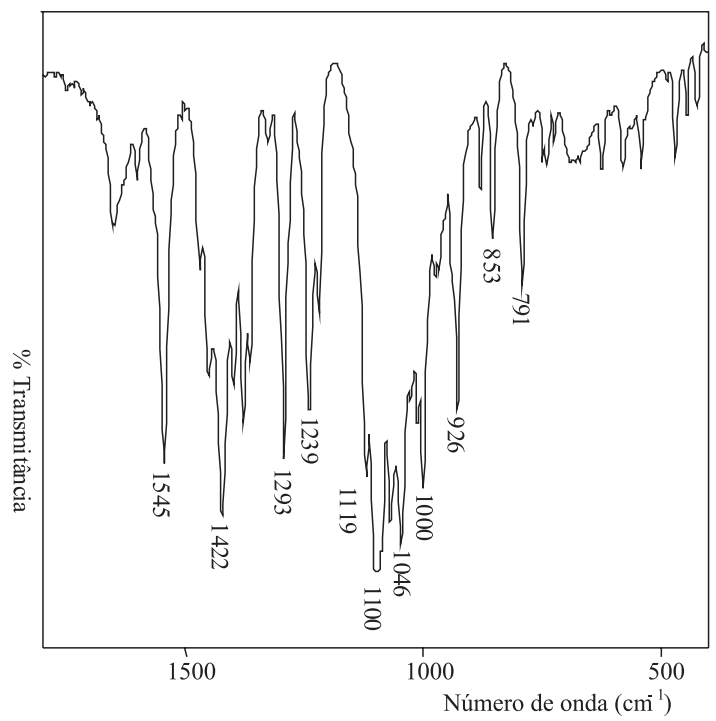

Figura 2. Espectro de infravermelho do boro-complexo com piridoxina

Para a obtenção dos espectros de RMN, prepara-se uma solução de $20 \mathrm{mg} / \mathrm{mL}$ do complexo isolado em $\mathrm{D}_{2} \mathrm{O}$.

Adquire-se um espectro de RMN de ${ }^{1} \mathrm{H}$. Apesar da existência de dois ligantes não idênticos no estado sólido (estrutura determinada por meio de difração de raios $\mathrm{X}$ em monocristal), ${ }^{13}$ o espectro da solução apresenta apenas um conjunto de picos (singletos) devido a uma rápida troca prototrópica. Identifique a origem dos sinais baseado nos valores relativos de integração. Adicionalmente aparece um pico de água residual em $\delta=4,8 \mathrm{ppm}$, referenciado internamente com DSS - sal sódico de ácido 3-(trimetilsilil)-1-propanossulfônico ( $\delta=$ 0 para o grupo metílico). O pico do grupo $\mathrm{OH}$ alcoólico encontra-se ocultado pelo sinal de $\mathrm{H}_{2} \mathrm{O}$.

Adquire-se um espectro de RMN de ${ }^{11} \mathrm{~B}$. Um único sinal de ressonância é observado em $\delta=1,77 \mathrm{ppm}$.

\section{Preparação de bis(oxalato)borato de potássio}

Uma mistura de $31,0 \mathrm{mg}(0,5 \mathrm{mmol})$ de ácido bórico, 94,5 mg $(0,75 \mathrm{mmol})$ de ácido oxálico di-hidratado e 46,0 $\mathrm{mg}(0,25 \mathrm{mmol})$ de oxalato de potássio mono-hidratado é triturada em um gral de porcelana e logo transferida para um balão de destilação de $100 \mathrm{~mL}$. Adiciona-se ao balão $50 \mathrm{~mL}$ de tolueno e uma barra magnética de agitação. Monta-se o equipamento de refluxo para separação contínua de água (dispositivo Dean Stark). A mistura é aquecida sobre um banho de óleo a $\pm 140{ }^{\circ} \mathrm{C}$, sob agitação vigorosa, e mantida sob refluxo até que o volume calculado de água tenha sido separado (aproximadamente $2 \mathrm{~h}$ ). Depois de esfriado à temperatura ambiente, o produto é filtrado sob vácuo utilizando um filtro de vidro sinterizado. Após seco na estufa a $120^{\circ} \mathrm{C}$ o resíduo sólido é pesado para determinação do o rendimento percentual (rendimento médio obtido $\mathrm{C}_{4} \mathrm{O}_{8} \mathrm{BK}$ : em torno de $90 \%$ ).

Registra-se um espectro de infravermelho (em pastilha de $\mathrm{KBr}$ ) e compara-se o mesmo com o espectro de referência (Figura 3). Verificase a ausência de materiais de partida no espectro e observam-se as frequências características do oxalatoborato $\left[\mathrm{B}\left(\mathrm{C}_{2} \mathrm{O}_{4}\right)_{2}\right]^{-}$. As bandas espectrais mais destacadas correspondem às vibrações de $v \mathrm{C}=\mathrm{O}(1816$ e $\left.1779 \mathrm{~cm}^{-1}\right), v$ B-O-C $\left(1304 \mathrm{~cm}^{-1}\right), v \mathrm{C}-\mathrm{C}\left(1219 \mathrm{~cm}^{-1}\right)$ e $v_{\mathrm{s}} \mathrm{BO}_{4}(1084$, o componente do isótopo ${ }^{10} \mathrm{~B}$ aparece como ombro em $\left.\left.1128 \mathrm{~cm}^{-1}\right)\right)^{4,5}$

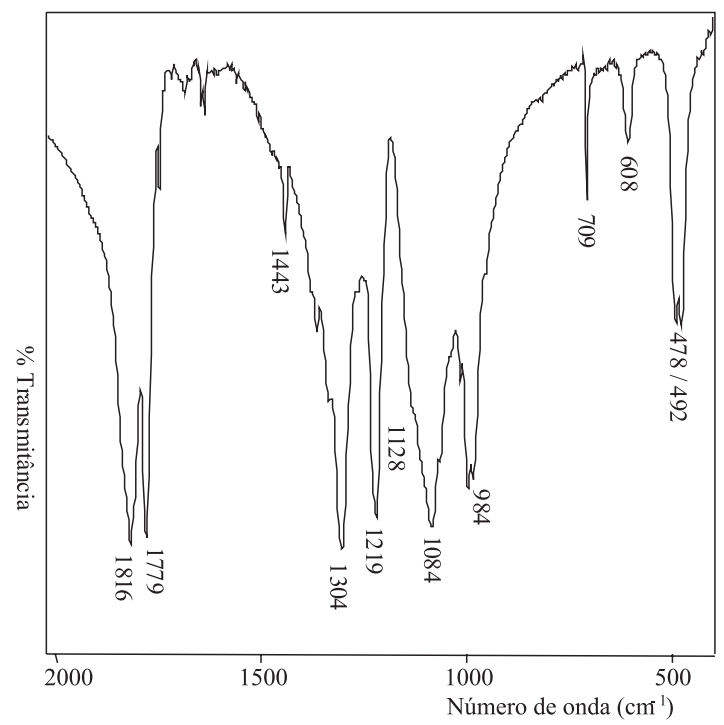

Figura 3. Espectro de infravermelho do oxalatoborato de potássio

Os espectros de RMN não podem ser obtidos, pois os oxalatoboratos somente são solúveis em água, onde são rapidamente hidrolisados em reversão de sua obtenção, conforme a reação 5 .

\section{CONCLUSÃO}

Os experimentos descritos foram realizados com êxito por alunos do $5^{\circ}$ semestre na disciplina "Laboratório de Química Inorgânica" dos cursos de graduação do Instituto de Química da Universidade 
de Brasília. A preparação dos complexos descritos envolve materiais baratos e facilmente acessíveis; a caracterização espectroscópica requerida para os propósitos desses experimentos pode ser alcançada na maioria dos centros de ensino e pesquisa do país.

\section{MATERIAL SUPLEMENTAR}

As Figuras 1S-8S que apresentam os espectros de infravermelho mais completos e os espectros de RMN dos compostos obtidos estão disponíveis em http://quimicanova.sbq.org.br, na forma de arquivo PDF, com acesso livre.

\section{AGRADECIMENTOS}

À FINEP pelo apoio financeiro (Projeto no 097001 CT-INFRA).

\section{REFERÊNCIAS}

1. Emry, J.; Györi, B. Em Comprehensive Coordination Chemistry; Wilkinson, G., ed.; Pergamon Press: Oxford, 1987, vol. 3, cap. 24.

2. Steinberg, H.; Brotherton, R.; Organoboron Chemistry, John Wiley: New York, 1964.

3. Heimann, W.; Sagredos, A. N.; Chem. Ber. 1965, 98, 1949.
4. Bessler, E.; Weidlein, J.; Z. Naturforsch. B 1982, 37, 1020.

5. Nakamoto, K.; Infrared and Raman Spectra of Inorganic and Coordination Compounds, $5^{\text {th }}$ ed., John Wiley \& Sons, Inc.: New York, 1997.

6. Weidlein, J.; Müller, U.; Dehnicke, K.; Schwingungsspektroskopie, Georg Thieme Verlag: Stuttgart, 1962.

7. Zanger, M.; Moyna, G.; J. Chem. Educ. 2005, 82, 1390.

8. Brevard, C.; Granger, P.; Handbook of High Resolution Multinuclear $N M R$, John Wiley \& Sons, Inc.: New York, 1981.

9. Wrackmeyer, B.; Tok, O. L.; Magn. Reson. Chem. 2002, 40, 406.

10. Hartman, J. S.; Schrobilgen G. J.; Can. J. Chem. 1972, 50, 713.

11. Brauer, G.; Handbook of Preparative Inorganic Chemistry, $3^{\text {rd }}$ ed., F. Enke Verlag: Stuttgart, 1978, vol. 1.

12. Scudi, J. V.; Bastedo, W. A.; Webb, T. J.; J. Biol. Chem. 1940, 136, 399.

13. Sousa, A. T.; Bessler, K. E.; Lemos, S. S.; Gomes, F. B.; Casagrande, G. A.; Lang, E. S.; Zeitschr. Anorg. Allg. Chem. 2007, 633, 771.

14. Brown, M. L.; US pat. 2,442,667. 1947. (CA 42, 5174).

15. Eaton, G. R.; Lipscomb, W. N.; NMR Studies of Boron Hydrides and Related Compounds, W.A. Benjamin, Inc.: New York, 1969.

16. Silverstein, R. M.; Webster, F. X.; Kiemle, D. J.; Spectrometric Identification of Organic Compounds, $7^{\text {th }}$ ed., John Wiley \& Sons, Inc.: New York, 2005, p. 326.

17. Brownstein, S.; Latremouille, G.; Can J. Chem. 1978, 56, 2764. 


\section{PREPARAÇÃO E CARACTERIZAÇÃO ESPECTROSCÓPICA DE COMPLEXOS DE BORO - UMA PROPOSTA PARA UMA PRÁTICA INTEGRADA DE QUÍMICA INORGÂNICA}

Karl Eberhard Bessler*, Adriana Torres de Sousa, Sebastião de Souza Lemos, Fernanda Batista Gomes e Elizeu José de Souza Instituto de Química, Universidade de Brasília, 70904-970 Brasília - DF, Brasil

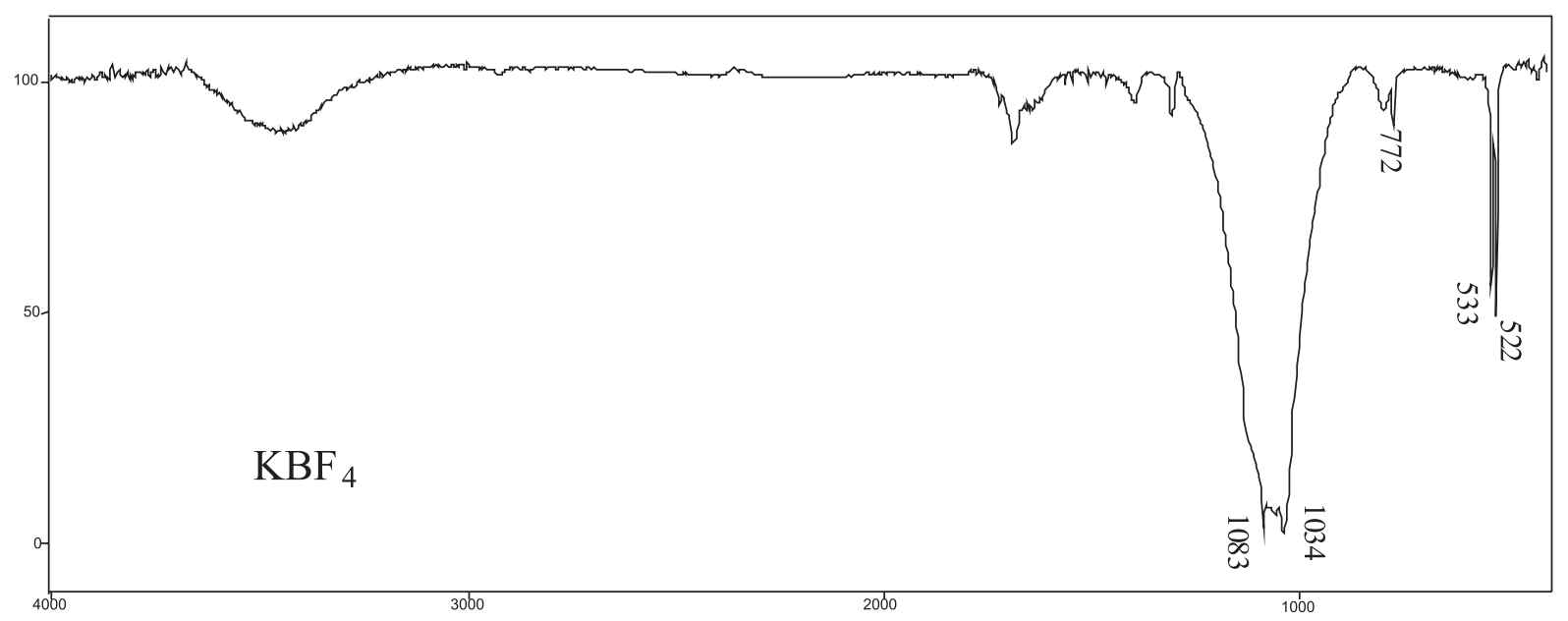

Figura 1S. Espectro de infravermelho de $\mathrm{KBF}_{4}$

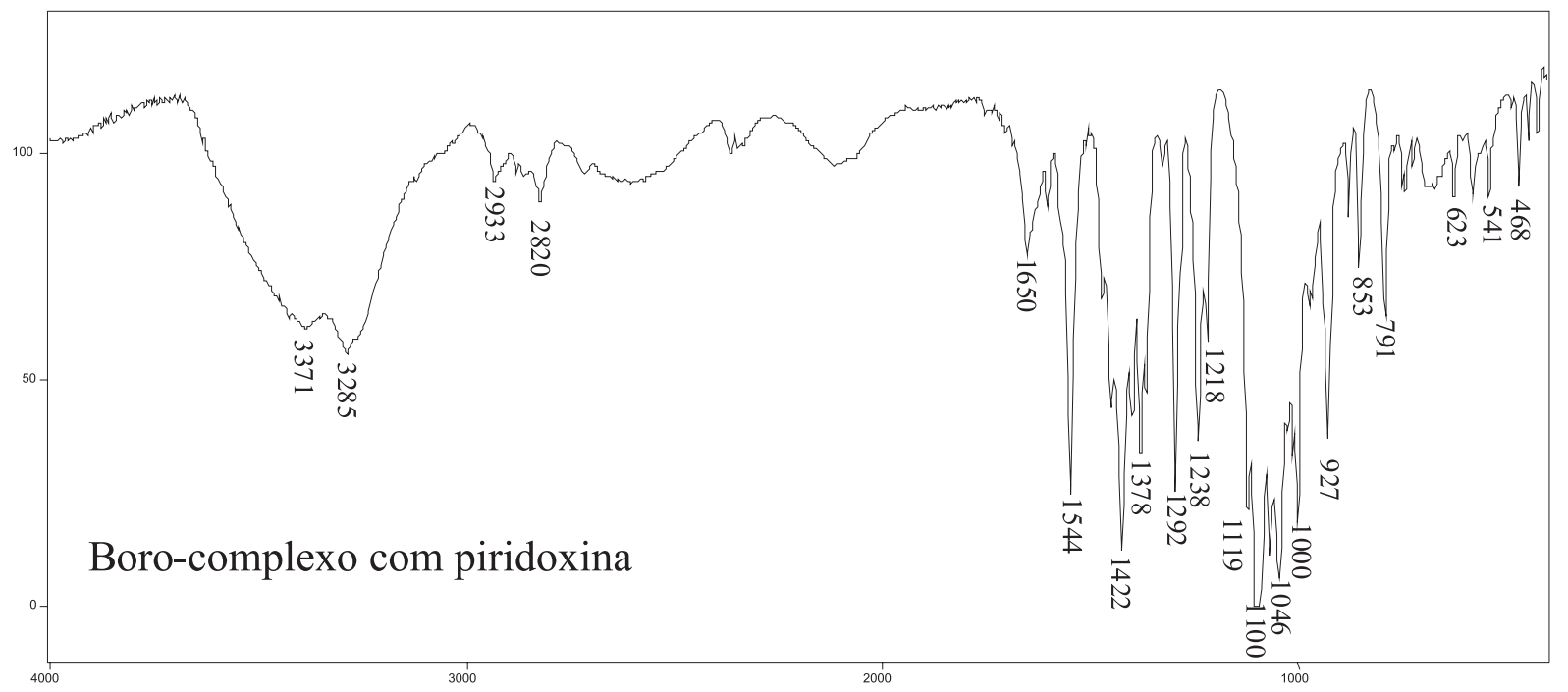

Figura 2S. Espectro de infravermelho do boro-complexo com piridoxina 


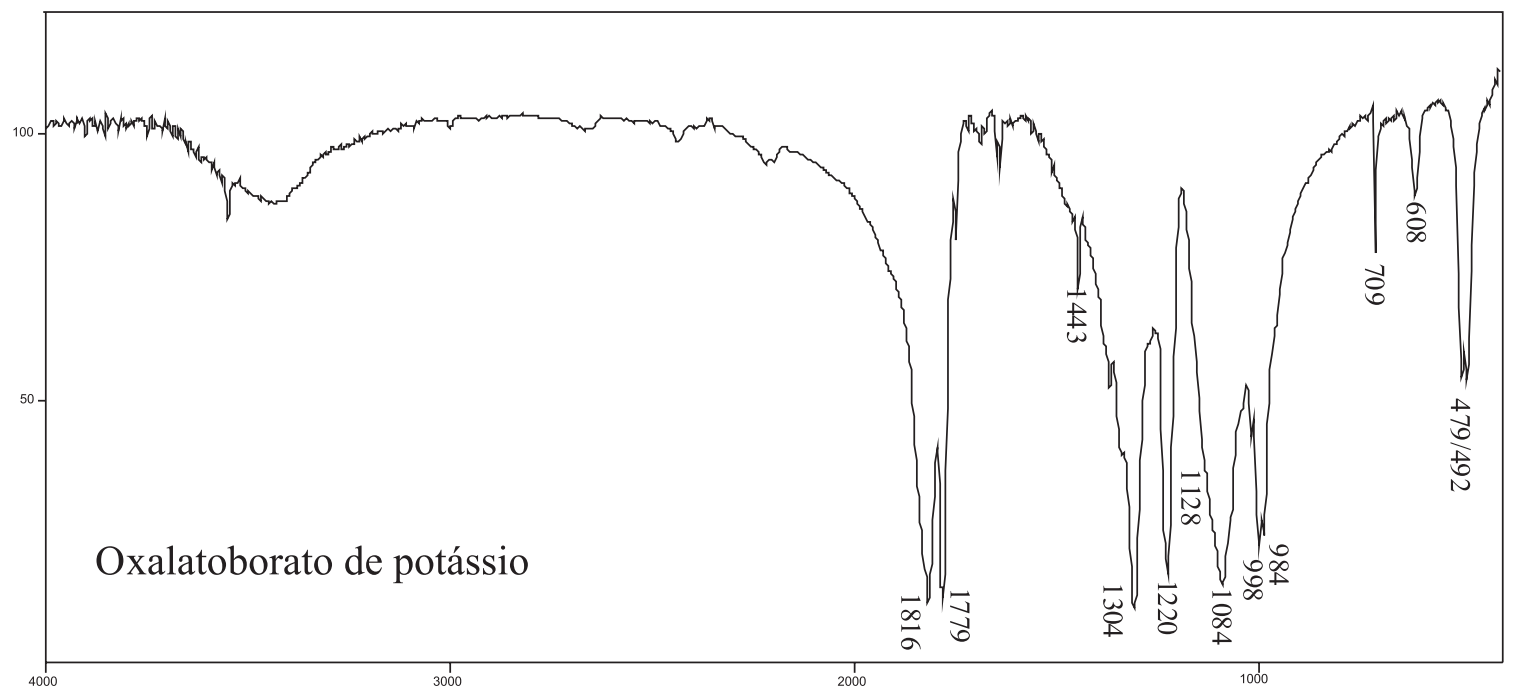

Figura 3S. Espectro de infravermelho do oxalatoborato de potássio

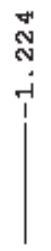

${ }^{11} \mathrm{~B}-\mathrm{RMN}$ de $\mathrm{KBF}_{4}$

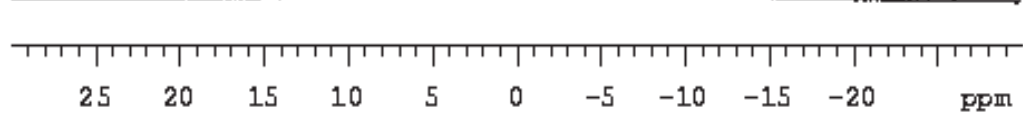

Figura 4S. Espectro de ${ }^{11} B-R M N$ de $K B F_{4}$

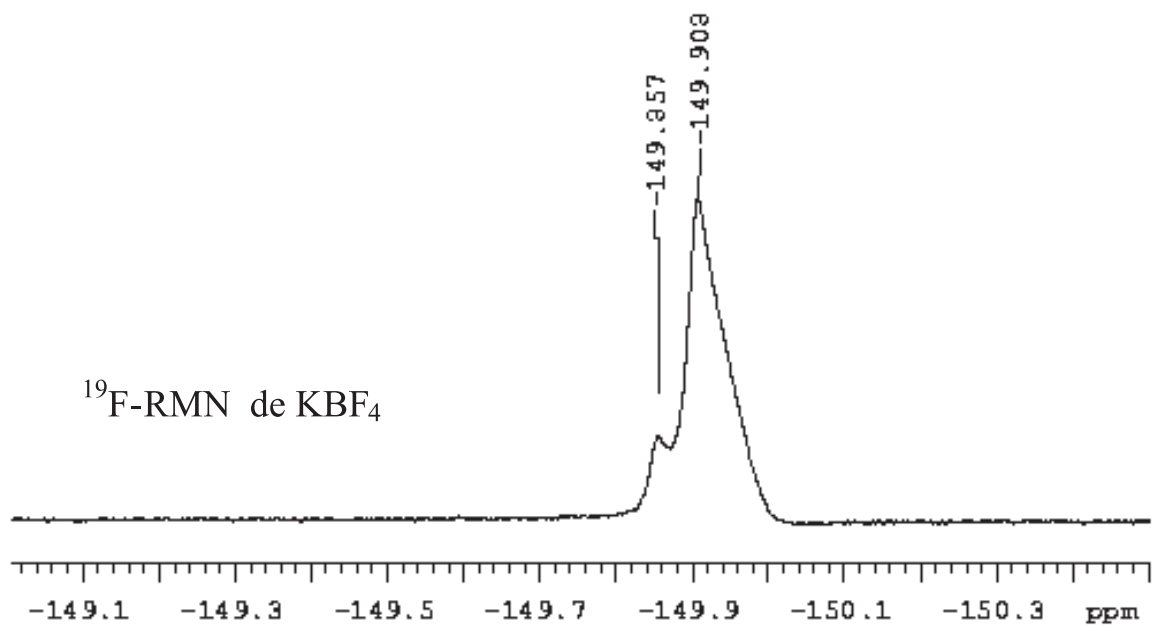

Figura 5S. Espectro de ${ }^{19} \mathrm{~F}-\mathrm{RMN}$ de $\mathrm{KBF}_{4}$ 


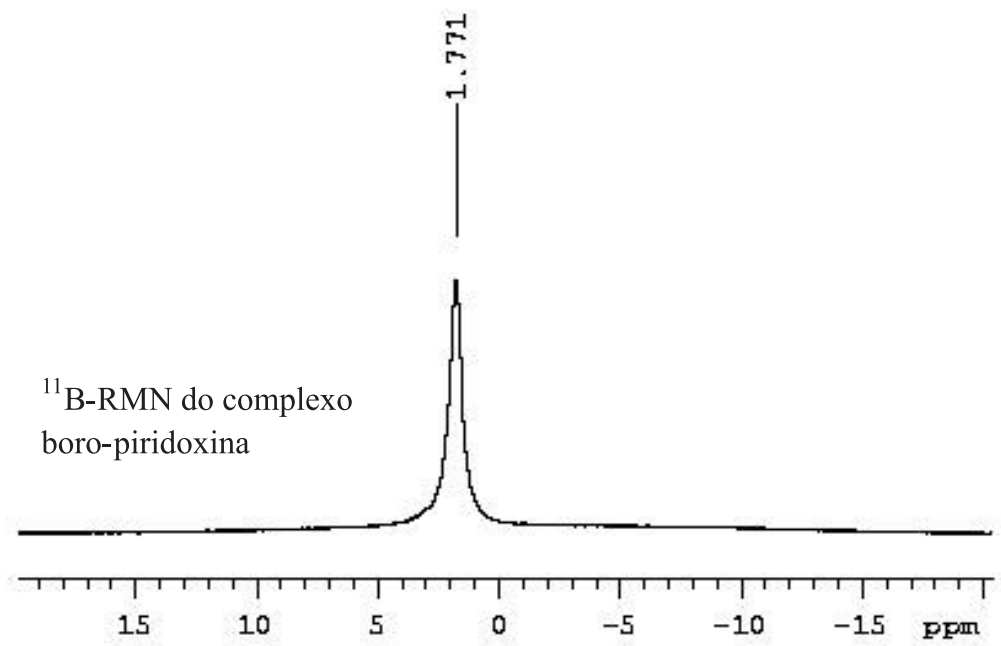

Figura 6S. Espectro de ${ }^{11} B-R M N$ do complexo boro-piridoxina

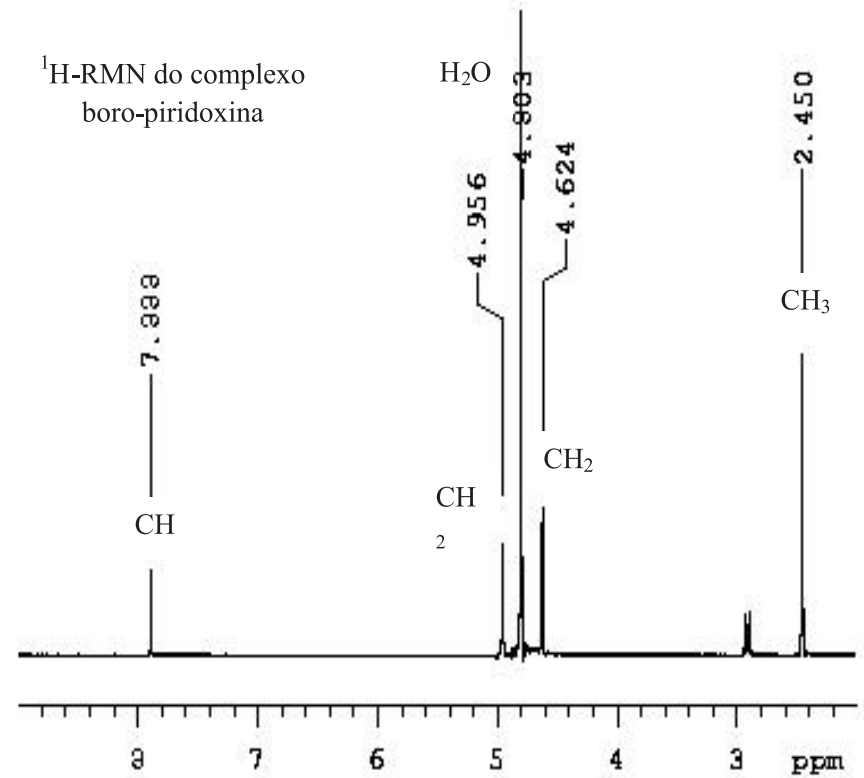

Figura 7S. Espectro de ${ }^{l} H$-RMN do complexo boro-piridoxina

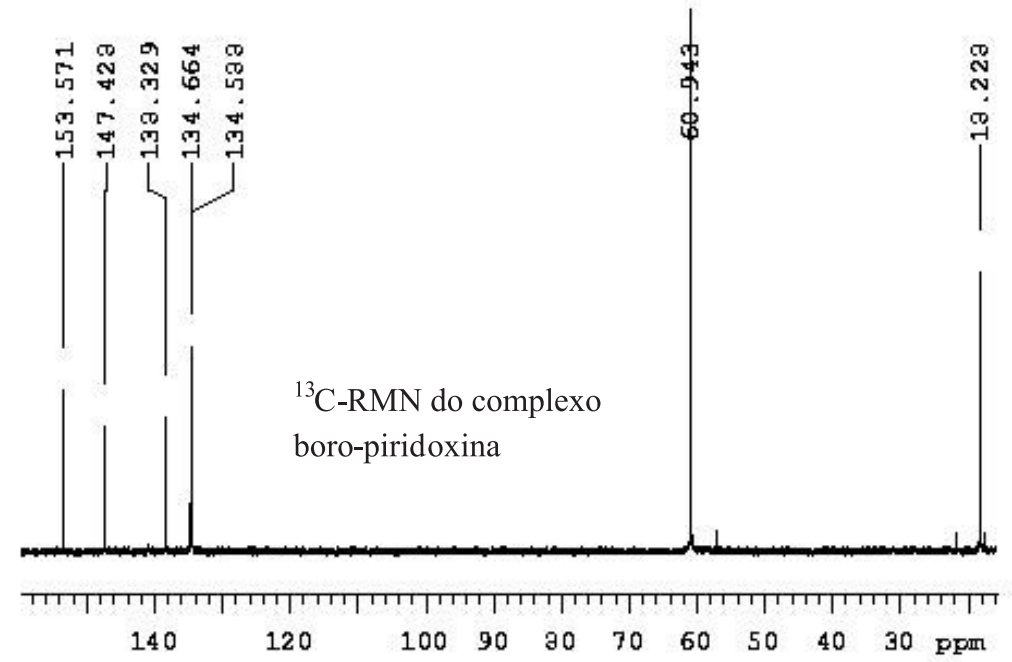

Figura 8S. Espectro de ${ }^{13} \mathrm{C}-\mathrm{RMN}$ do complexo boro-piridoxina 